Supporting Information for

\title{
Informatics view on the challenges of identifying missing proteins from shotgun
}

\section{proteomics}

Wai-Kok Choong ${ }^{\ddagger}, 1$, Hui-Yin Chang ${ }^{\ddagger}{ }^{\ddagger 1,2,3}$, Ching-Tai Chen ${ }^{\ddagger}, 1$, Chia-Feng Tsai ${ }^{4}$, Wen-Lian Hsu ${ }^{1}$, Yu-Ju Chen ${ }^{4, *}$, Ting-Yi Sung ${ }^{1, *}$

${ }^{1}$ Institute of Information Science, Academia Sinica, Taipei 11529, Taiwan; ${ }^{2}$ Bioinformatics Program, Taiwan International Graduate Program, Academia Sinica, Taipei 11529, Taiwan; ${ }^{3}$ Institute of Biomedical Informatics, National Yang-Ming University, Taipei 11221, Taiwan; ${ }^{4}$ Institute of Chemistry, Academia Sinica, Taipei, Taiwan

* Corresponding author:

Ting-Yi Sung

Email: tsung@iiis.sinica.edu.tw

Phone: +886-2-2788-3799

Fax number: +886-2-2782-4814

Yu-Ju Chen

E-mail: yujuchen@gate.sinica.edu.tw

Phone: +886-2-27898660

Fax number: +886-2-27831237

\section{Contents}

Figure S-1. The distributions of unique and shared peptides of different lengths based on in silico Lys-C digestion. 3

Figure S-2. The distributions of unique and shared peptides of different lengths based on in silico trypsin and Lys-C tandem digestion. 4

Figure S-3. The Venn diagram of digested peptides and 20,053 proteins containing at least one unique digested peptide yielded by trypsin, Lys-C and both enzymes, when peptide length is limited to 6-40 amino acids......... 5 Figure S-4. The distributions of proteins with different numbers of unique peptides using trypsin, Glu-C, Lys-N, and Lys-C, respectively. 6

Figure S-5. The distribution of protein hydrophobicity (in terms of Gravy value) in ten families and domains classified by InterPro..

Figure S-6. The distribution of peptide hydrophobicity (in terms of Gravy value) in ten families and domains classified by InterPro. 9

Figure S-7. The distribution of protein length in ten families and domains classified by InterPro. 
Figure S-8. The distribution of unique peptide length in ten families and domains classified by InterPro..........11

Figure S-9. The distribution of unique peptide number in ten families and domains classified by InterPro....... 12 


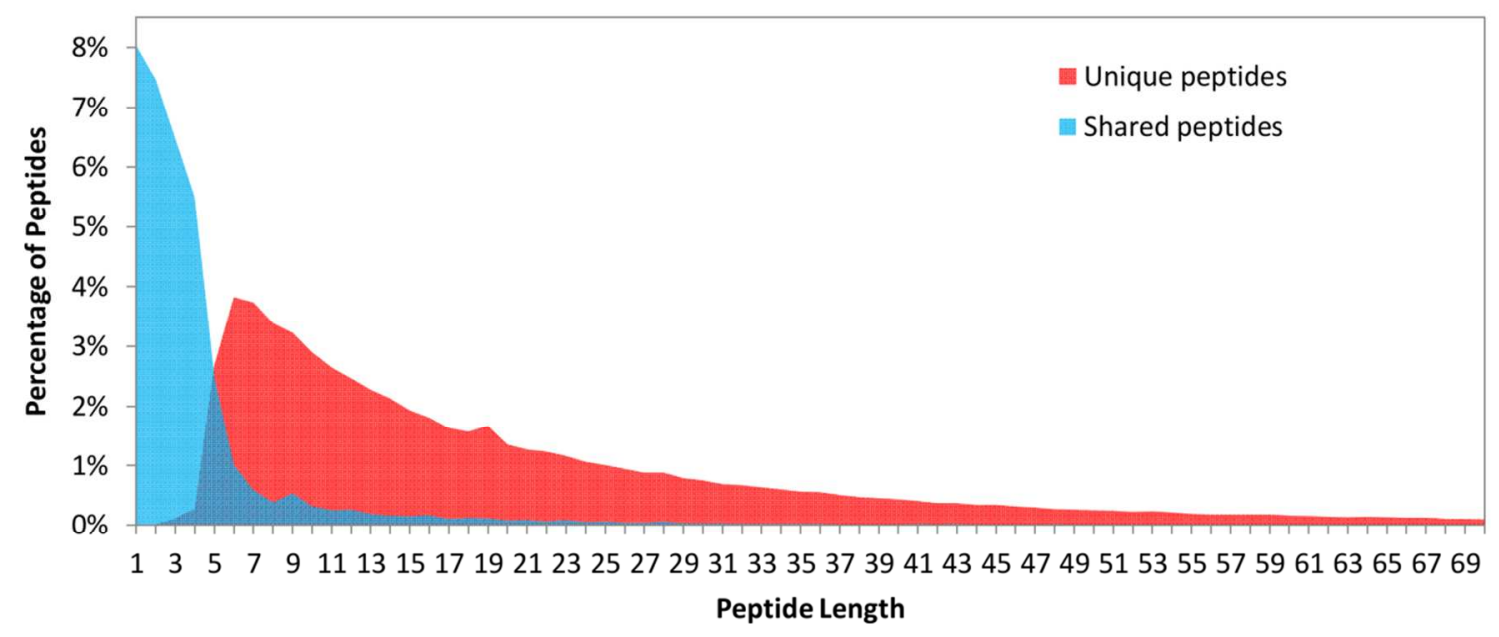

Figure S-1. The distributions of unique and shared peptides of different lengths based on in silico Lys-C digestion. Red and blue areas represent the distributions of unique and shared peptides, respectively, regarding to different peptide lengths shown in the X-axis. The numbers of unique and shared peptides were 431,157 and 235,285 , respectively. The data was derived from in silico trypsin digestion of 20,189 human proteins in UniProt. 


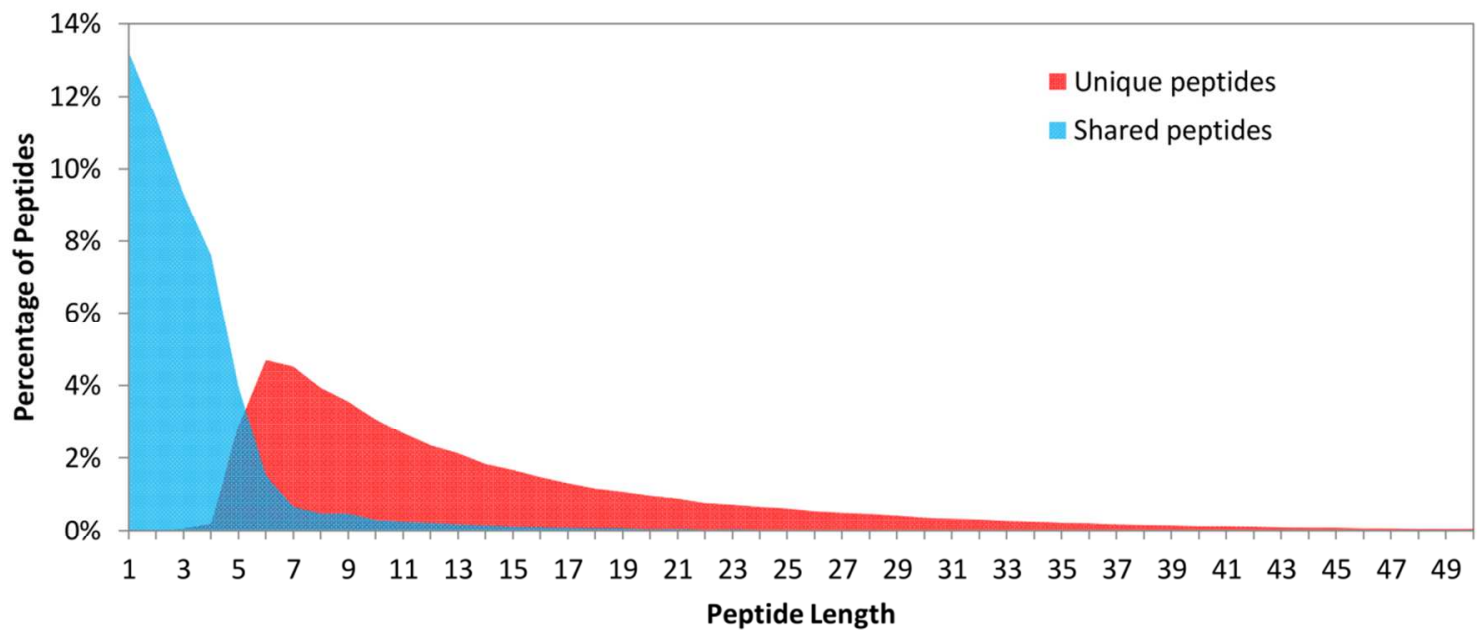

Figure S-2. The distributions of unique and shared peptides of different lengths based on in silico trypsin and Lys-C tandem digestion. Red and blue areas represent the distributions of unique and shared peptides, respectively, regarding to different peptide lengths shown in the $\mathrm{x}$-axis. The numbers of unique and shared peptides were 621,501 and 639,361, respectively. The data was derived from in silico trypsin digestion of 20,189 human proteins in UniProt. 

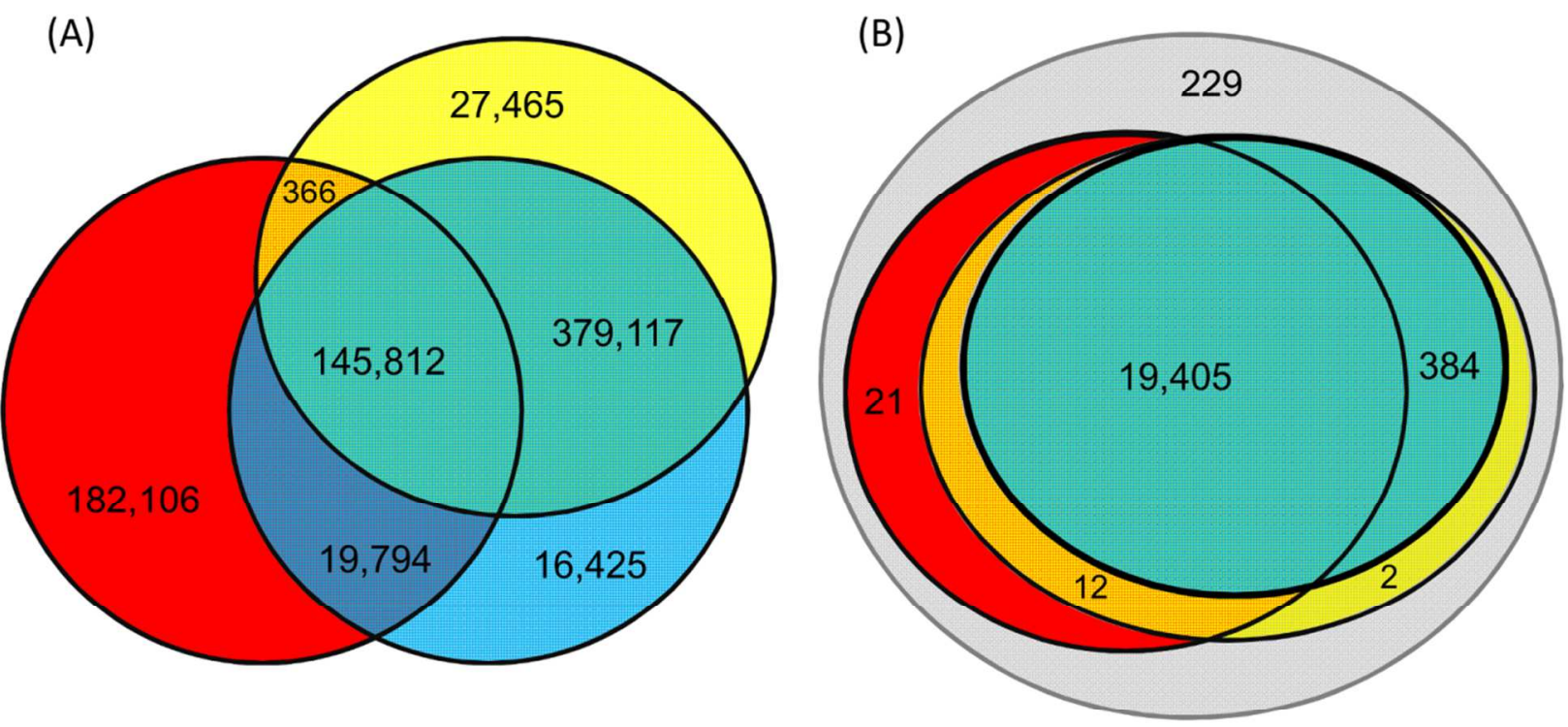

\section{OTrypsin OLys-C OTrypsin+Lys-C O No Unique Peptide}

Figure S-3. The Venn diagram of digested peptides and 20,053 proteins containing at least one unique digested peptide yielded by trypsin, Lys-C and both enzymes, when peptide length is limited to 6-40 amino acids. (A) The number of non-redundant unique peptides with amino acid length ranging from 6 to 40 using trypsin, Lys-C, and trypsin+Lys-C digestion. A number of 16,425 unique peptides are additionally obtained using trypsin+Lys-C digestion whereas 27,465 and 182,106 unique peptides are additionally obtained using trypsin and Lys-C, respectively. (B) The number of proteins with at least one non-redundant unique peptide of which the sequence range is 6 to 40 amino acids. In silico digestion analysis was conducted on 20,053 proteins commonly in UniProt and neXtProt. Among the 20,053 proteins, 19,405 proteins had at least one unique peptide from any of the three digestions, and 229 proteins did not have any unique peptide. 
(A)

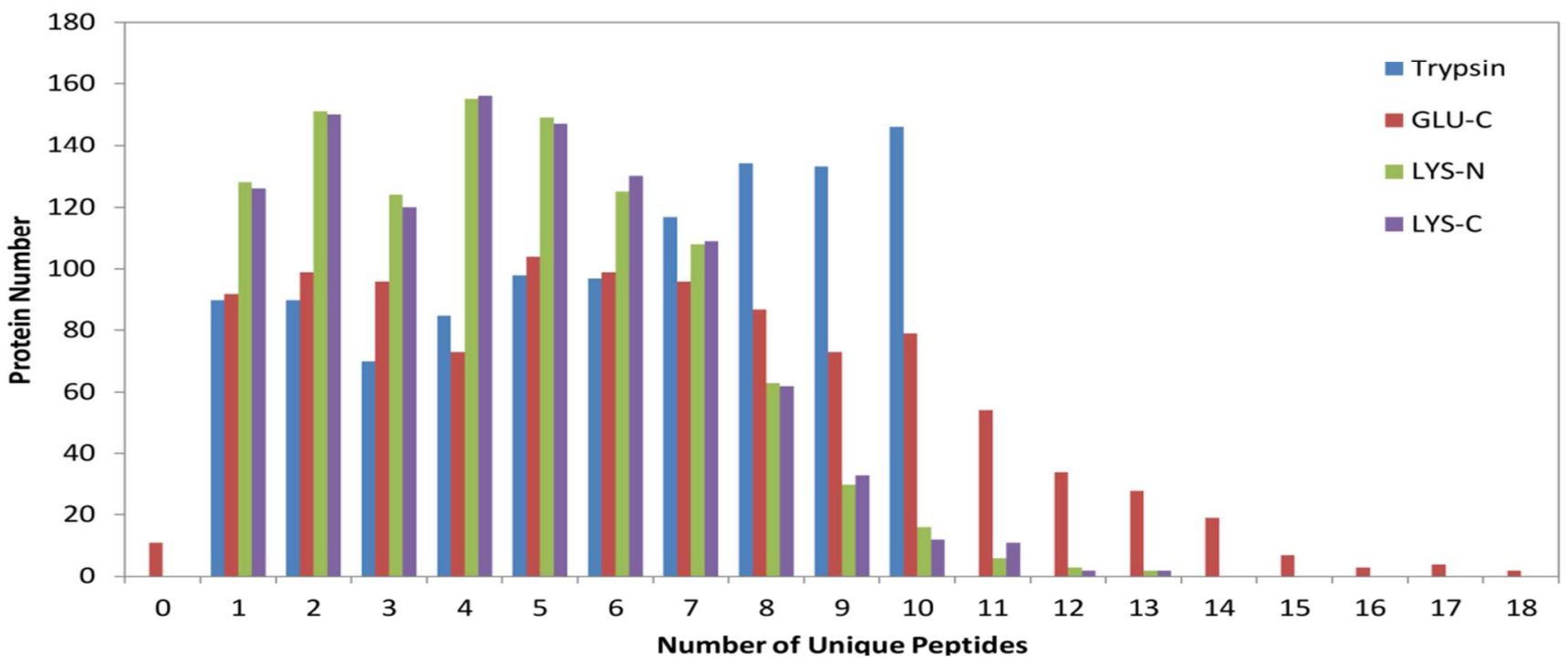

(B)

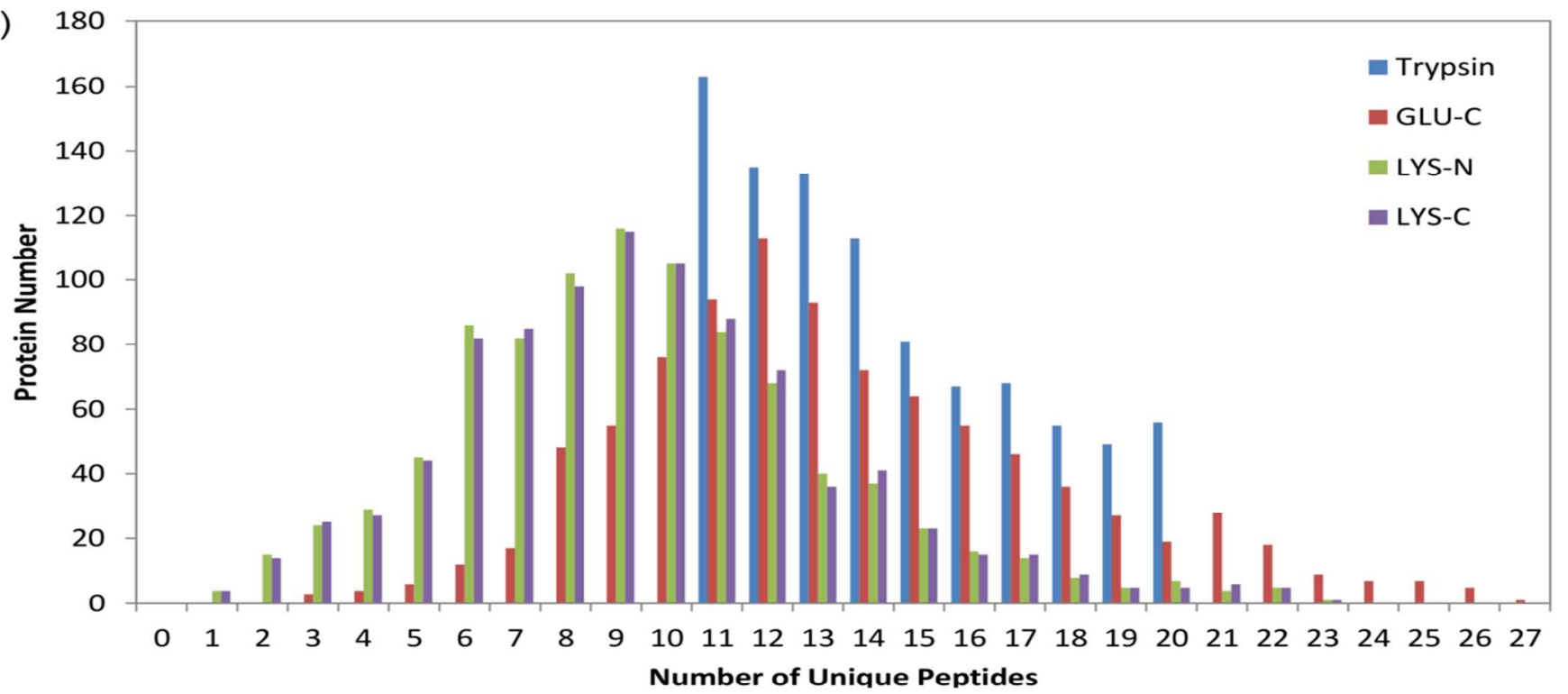

(C)

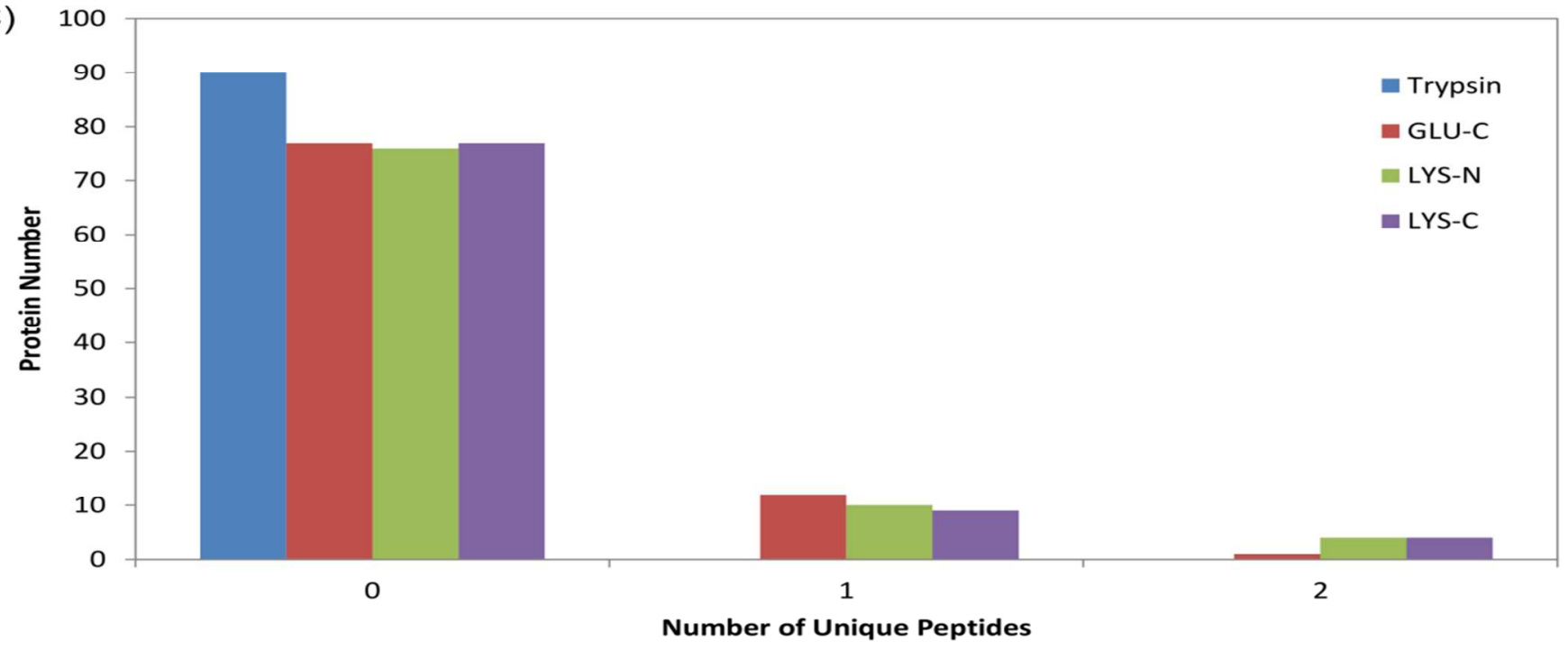

Figure S-4. The distributions of proteins with different numbers of unique peptides using trypsin, Glu-C, Lys-N, and Lys-C, respectively. The distributions of the numbers of unique peptides in 2,070 missing proteins 
were digested by trypsin, Glu-C, Lys-N, and Lys-C, respectively. This in silico analysis was conducted on missing proteins with at most 20 unique tryptic peptides. These proteins were divided into three groups according to the number of unique tryptic peptides: proteins with 1-10 unique tryptic peptides (Group 1), with 11-20 unique tryptic peptides (Group 2), and without any unique tryptic peptide (Group 3). The distributions of proteins in the three groups were shown in $\mathrm{A}, \mathrm{B}$ and $\mathrm{C}$, respectively. 


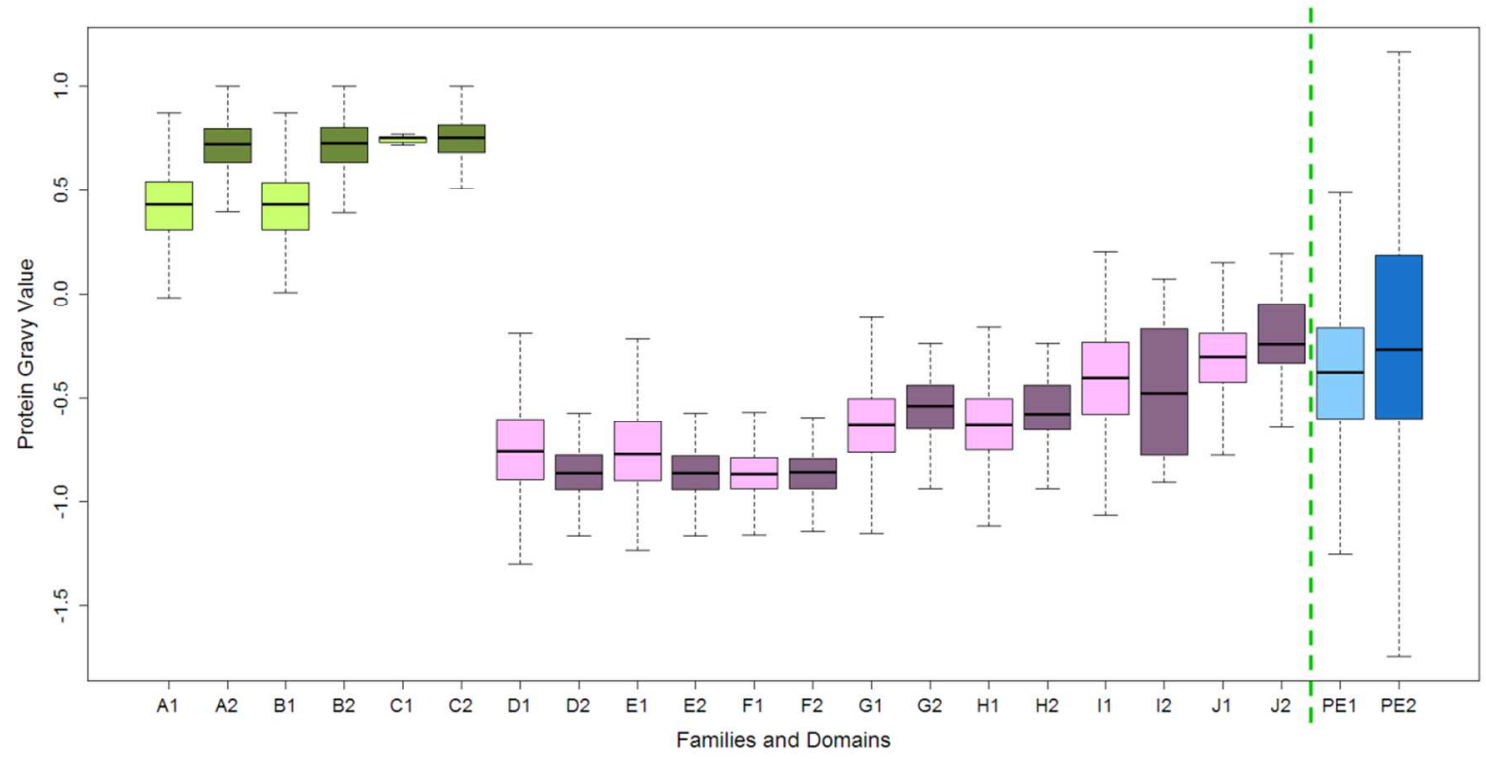

Figure S-5. The distribution of protein hydrophobicity (in terms of Gravy value) in ten families and domains classified by InterPro. The ten families or domains containing the most number of proteins with PE2 evidence were included in this analysis; they are A: IPR017452 (GPCR, rhodopsin-like, 7TM), B: IPR000276 (G protein-coupled receptor, rhodopsin-like), C: IPR000725 (Olfactory receptor), D: IPR015880 (Zinc finger, C2H2-like ), E: IPR013087 (Zinc finger C2H2-type/integrase DNA-binding domain), F: IPR001909 (Krueppel-associated box), G: IPR009057 (Homeodomain-like), H: IPR001356 (Homeobox domain (IPR001356)), I: IPR020683 (Ankyrin repeat-containing domain), and J: IPR013783 (Immunoglobulin-like fold). Family/domain X1 and X2 denotes proteins with PE1 and PE2 evidences, respectively. In addition, Group PE1 and PE2 represent the total 16,490 and 2,646 proteins with evidences at PE1 and PE2 levels, respectively. 


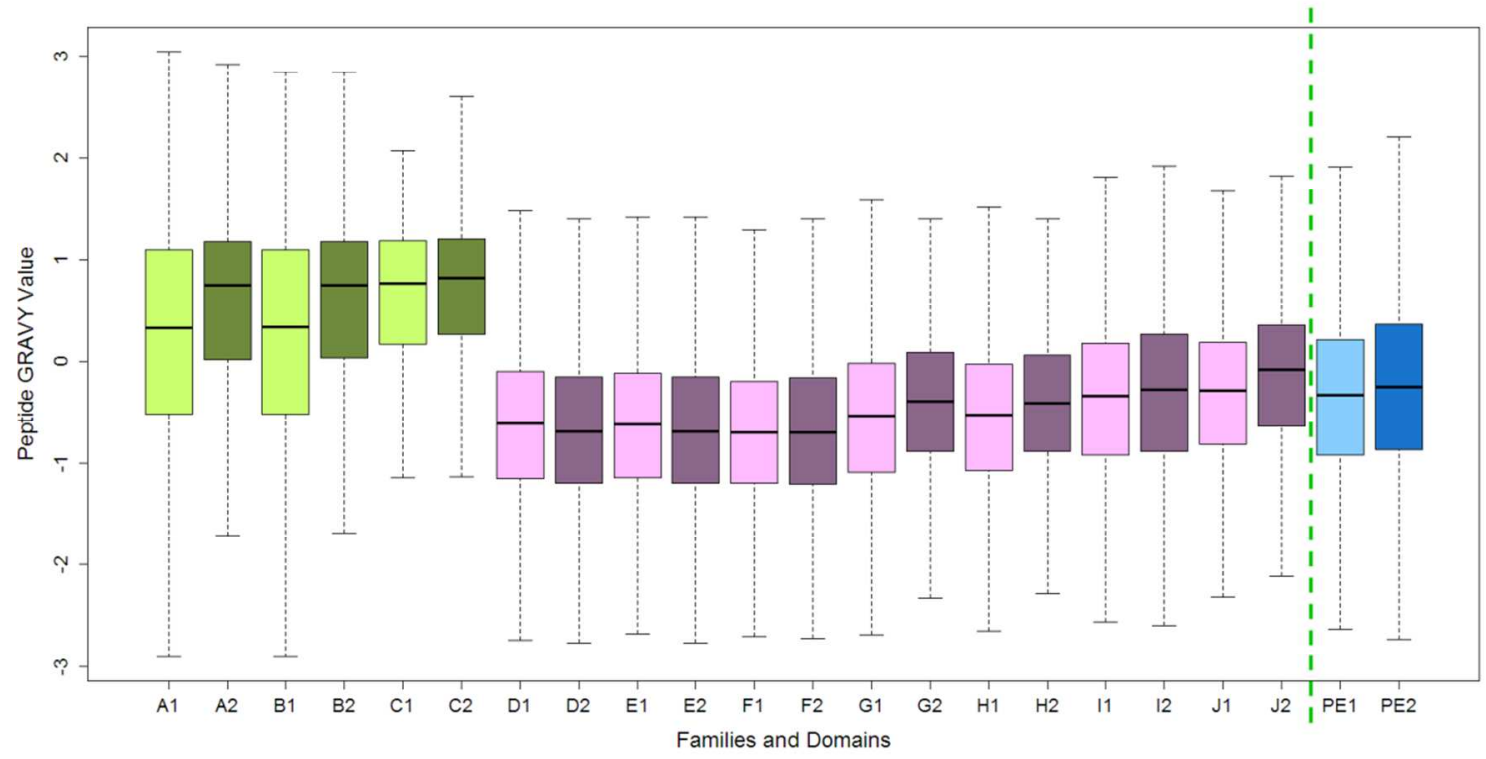

Figure S-6. The distribution of peptide hydrophobicity (in terms of Gravy value) in ten families and domains classified by InterPro. The ten families or domains containing the most number of proteins with PE2 evidence were included in this analysis; they are A: IPR017452 (GPCR, rhodopsin-like, 7TM), B: IPR000276 (G protein-coupled receptor, rhodopsin-like), C: IPR000725 (Olfactory receptor), D: IPR015880 (Zinc finger, C2H2-like), E: IPR013087 (Zinc finger C2H2-type/integrase DNA-binding domain), F: IPR001909 (Krueppel-associated box), G: IPR009057 (Homeodomain-like), H: IPR001356 (Homeobox domain (IPR001356)), I: IPR020683 (Ankyrin repeat-containing domain), and J: IPR013783 (Immunoglobulin-like fold). Family/domain X1 and X2 denotes proteins with PE1 and PE2 evidences, respectively. In addition, Group PE1 and PE2 represent the total 16,490 and 2,646 proteins with evidences at PE1 and PE2 levels, respectively. 


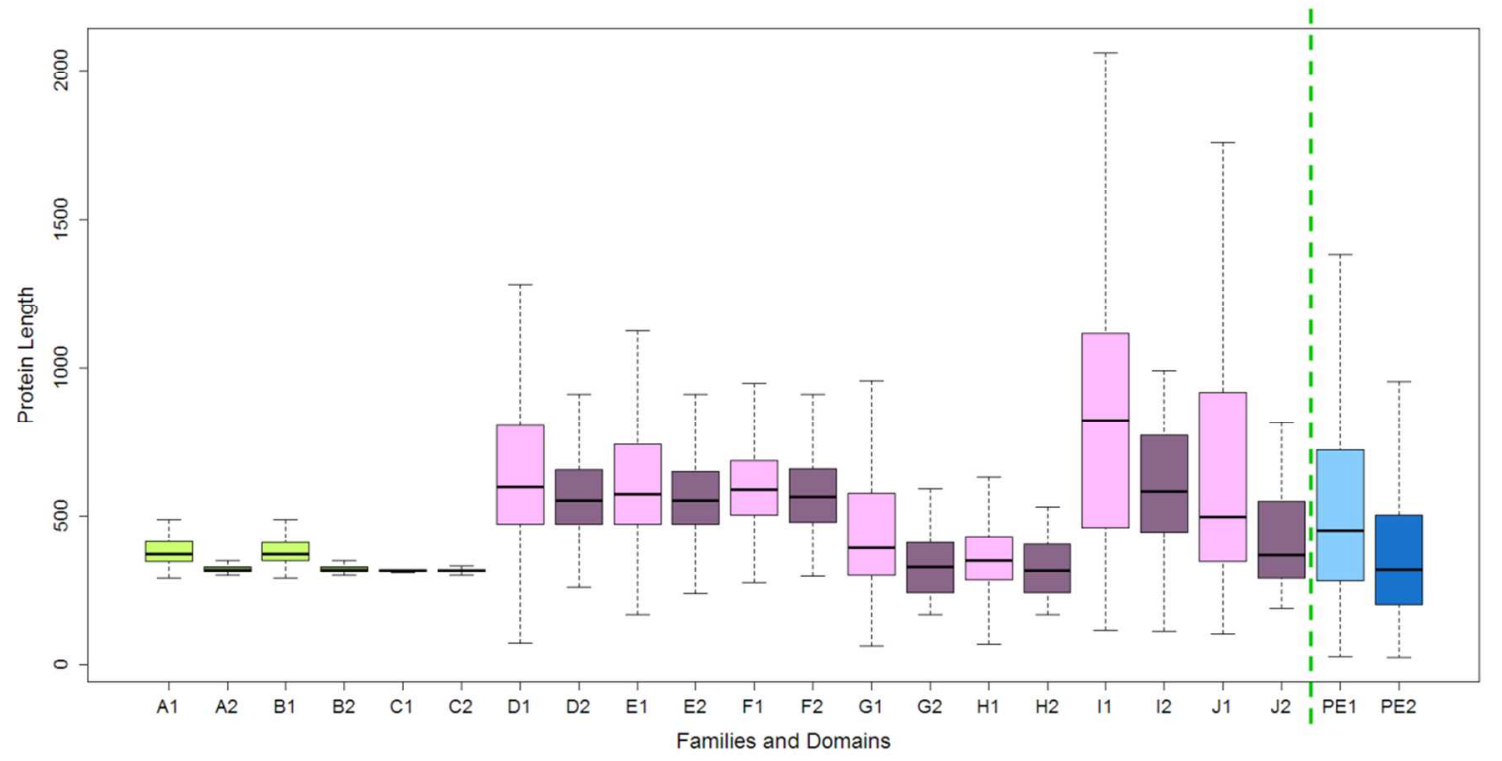

Figure S-7. The distribution of protein length in ten families and domains classified by InterPro. The ten families or domains containing the most number of proteins with PE2 evidence were included in this analysis; they are A: IPR017452 (GPCR, rhodopsin-like, 7TM), B: IPR000276 (G protein-coupled receptor, rhodopsin-like), C: IPR000725 (Olfactory receptor), D: IPR015880 (Zinc finger, C2H2-like ), E: IPR013087 (Zinc finger C2H2-type/integrase DNA-binding domain), F: IPR001909 (Krueppel-associated box), G: IPR009057 (Homeodomain-like), H: IPR001356 (Homeobox domain (IPR001356)), I: IPR020683 (Ankyrin repeat-containing domain), and J: IPR013783 (Immunoglobulin-like fold). Family/domain X1 and X2 denotes proteins with PE1 and PE2 evidences, respectively. In addition, Group PE1 and PE2 represent the total 16,490 and 2,646 proteins with evidences at PE1 and PE2 levels, respectively. 


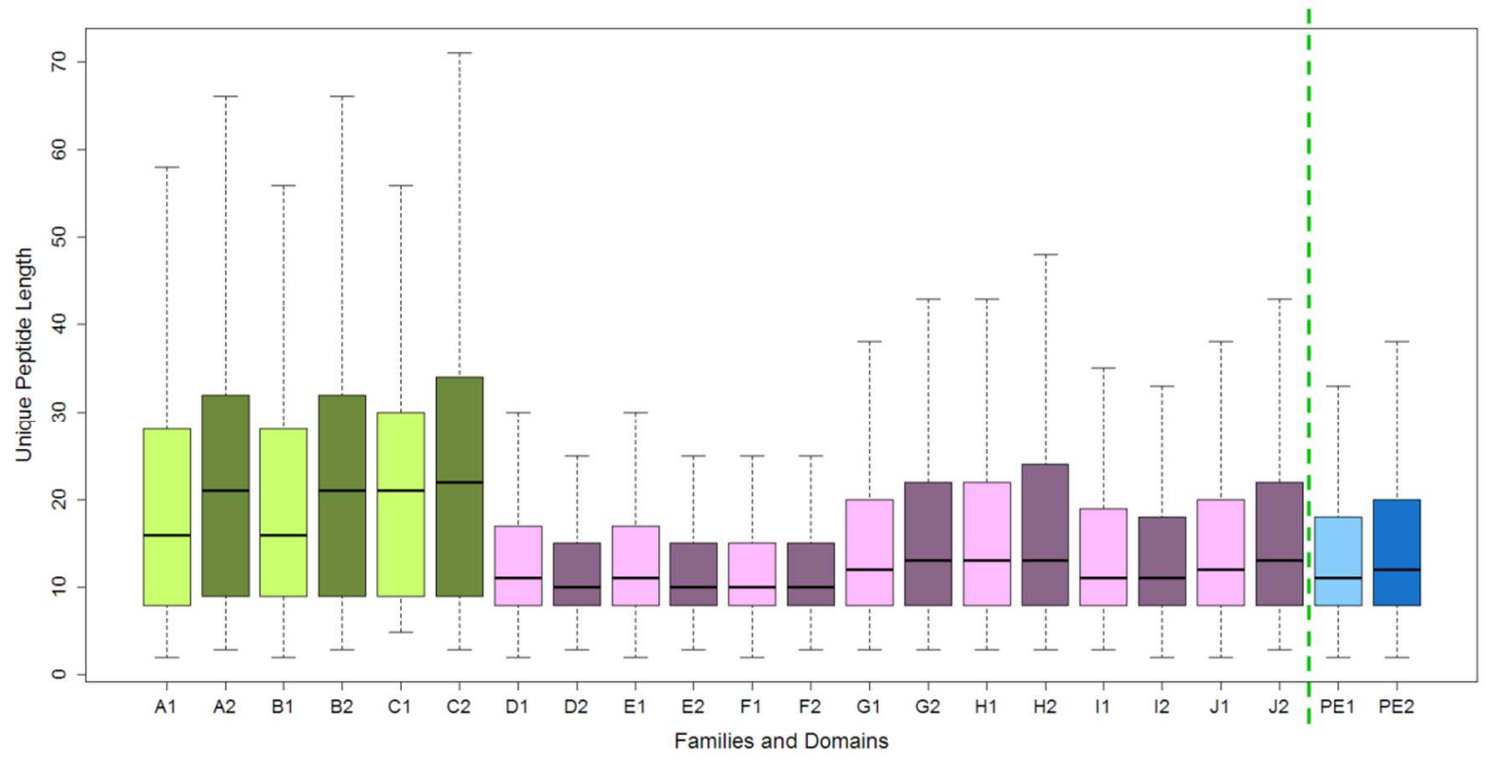

Figure S-8. The distribution of unique peptide length in ten families and domains classified by InterPro. The ten families or domains containing the most number of proteins with PE2 evidence were included in this analysis; they are A: IPR017452 (GPCR, rhodopsin-like, 7TM), B: IPR000276 (G protein-coupled receptor, rhodopsin-like), C: IPR000725 (Olfactory receptor), D: IPR015880 (Zinc finger, C2H2-like ), E: IPR013087 (Zinc finger C2H2-type/integrase DNA-binding domain), F: IPR001909 (Krueppel-associated box), G: IPR009057 (Homeodomain-like), H: IPR001356 (Homeobox domain (IPR001356)), I: IPR020683 (Ankyrin repeat-containing domain), and J: IPR013783 (Immunoglobulin-like fold). Family/domain X1 and X2 denotes proteins with PE1 and PE2 evidences, respectively. In addition, Group PE1 and PE2 represent the total 16,490 and 2,646 proteins with evidences at PE1 and PE2 levels, respectively. 


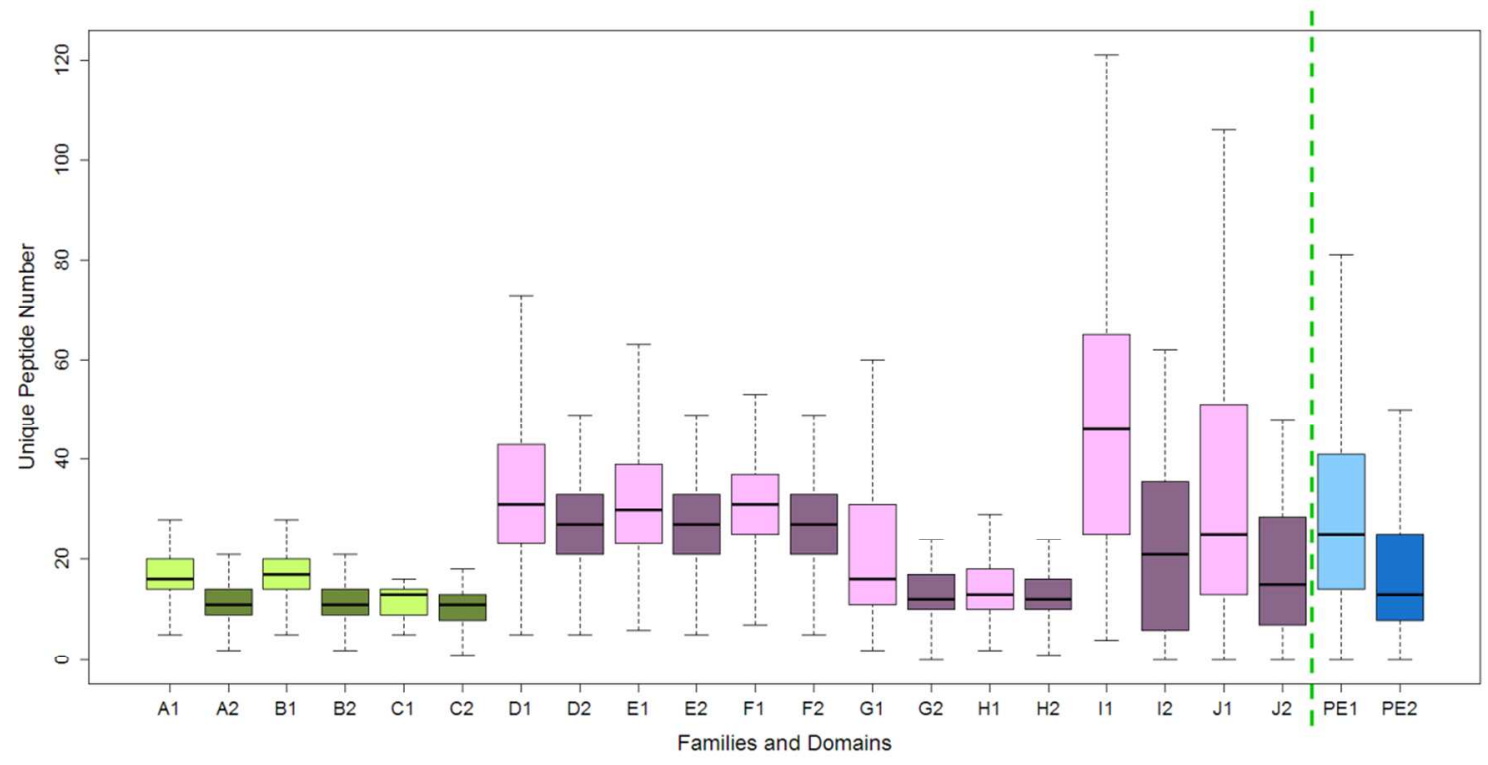

Figure S-9. The distribution of unique peptide number in ten families and domains classified by InterPro. The ten families or domains containing most number of proteins with PE2 evidence were included in this analysis; they are A: IPR017452 (GPCR, rhodopsin-like, 7TM), B: IPR000276 (G protein-coupled receptor, rhodopsin-like), C: IPR000725 (Olfactory receptor), D: IPR015880 (Zinc finger, C2H2-like ), E: IPR013087 (Zinc finger C2H2-type/integrase DNA-binding domain), F: IPR001909 (Krueppel-associated box), G: IPR009057 (Homeodomain-like), H: IPR001356 (Homeobox domain (IPR001356)), I: IPR020683 (Ankyrin repeat-containing domain), and J: IPR013783 (Immunoglobulin-like fold). Family/domain X1 and X2 denotes proteins with PE1 and PE2 evidences, respectively. In addition, Group PE1 and PE2 represent the total 16,490 and 2,646 proteins with evidences at PE1 and PE2 levels, respectively. 